\title{
Dissolution testing as a quality control tool during scale-up of immediate release oral, solid dosage forms.
}

Gienn A. Van Buskirk, Ph.D., Pbarmaceutical Division, Ciba-Geigy Corp., Summit, N7

\section{Alstract}

Procedures to evaluate and control the reproducibility of oral, solid dosage forms during batch scale-up are receiving considerable regulatory attention and are clearly an area of interest to pharmaceutical scientists. One important quality control tool in use by pharmaceutical scientists to monitor the scale-up of oral, solid dosage forms is dissolution testing.

A dissolution procedure is important at all stages of product development and scale-up. Dissolution testing may be useful in optimizing the formula or manufacturing process for an oral, solid dosage form during early development. Later in development, dissolution can be used to show reproducibility of dosage form performance during batch scale-up or as a result of process modifications or manufacturing site changes. The utility of dissolution testing, long regarded by both the USP and FDA as an effective quality control tool, has gained increased attention through its incorporation into the FDA's Interim Guidance: Immediate Release Solid Oral Dosage Forms, Pre- and Post-Approval Changes: Chemistry, Manufacturing and Controls, In Vitro Dissolution Testing, and In Vivo Bioequivalence Documentation[1]. This document allows the pharmaceutical scientist to use a discriminating dissolution method to show 'sameness' of a manufacturing process or formula change during scale-up. This tool opens the door to defining any such qualified change as "minor" and expedites the change approval process.

\section{Intraduction}

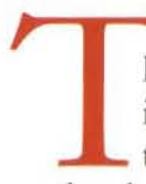
he use of dissolution testing as a quality control tool grew explosively in the decade of the 1970s. During the ' 80 s the pharmaceutical industry began to develop a data base that connected the dissolution performance of oral, solid dosage forms with their bioavailability/bioequivalence. Today, the existing tools allow pharmaceutical scientists to relate dissolution with the pharmacokinetic properties of many drug products. An approach, known as deconvolution of the plasma time course profile may permit the estimation of in-vivo dissolution. Thus, a point-to-point correlation of in-vitro and in-vivo dissolution may be developed. When such a correlation can be established, an invaluable evaluation tool becomes immediately available for an expanded role.

The value of dissolution testing was discussed extensively at two scale-up workshops composed of pharmaceutical scientists representing academe, government, and industry. The reports from these workshops which dealt with scale-up considerations for conventional oral, solid dosage forms and extended release oral forms have been published extensively in the US[2-7] and Europe[8,9]. The content of the first report which dealt with conventional, oral solid dosage forms was subjected to further scrutiny through FDA-sponsored extra-mural studies. These studies tested the bioavailability of a series of tablets manufactured with different formulation, scale and process variables. The results led to the publication of the interim guidance (see reference 1) on conventional oral, solid dosage forms and is expected to lead to a similar guidance on extended release dosage forms.

\section{Diseussion: Rationale for Dissolution Testing During Scale-Up}

The dissolution properties (extent and profile) of a finished dosage form should be monitored during product scale-up. Assuming that a meaningful, robust dissolution procedure has been developed, it can be a powerful tool to evaluate the impact of formula, process, equipment and site changes that may occur during product scale-up. The definition of 'robust' in this context is that of a procedure which has all of the classical analytical properties of linearity, precision, reproducibility and accuracy. A good method will employ a dissolution medium that is physiologically relevant (meaningful) in terms of volume and composition and 


\section{Quality Control....continued}

will be conducted using a currently accepted USP apparatus and standards (e.g., provide adequate sink conditions).

The AAPS workshop proposed an additional issue for consideration during selection of a dissolution method. The Workshop Report suggested that drugs be separated into one of three categories based on the understanding of the drug's solubility and permeability. The recommendation was to classify drugs as 1) highly permeable/ highly soluble 2) highly permeable/poorly soluble; poorly permeable/highly soluble or 3) poorly permeable/poorly soluble. A somewhat arbitrary definition of solubility was established based on the typical volume ingested at the time a dosage form is administered, e.g., $250 \mathrm{~mL}$. If the total dose of drug is soluble in $<250 \mathrm{~mL}$, it is considered highly soluble. Drugs with an extent of absorption of $>90 \%$ in the intestinal tract are considered to be highly permeable. Subsequent to the Workshop Report, additional clarity on this topic was provided in an article which describes the theoretical basis of this approach [10].

Using these definitions it is safe to assume that drugs that satisfy category 1 are not subject to bioavailability issues and their dissolution profile is expected to be reflected in complete and rapid absorption and bioavailability. For such drugs, a single point dissolution specification
( $Q$ value) of $85 \%$ in 30 minutes should be an adequate quality control check. For category 1 drugs, changes in formulation, manufacturing process or other issues (e.g., site change) can be monitored through characterization of the dissolution profile and are considered to be 'minor'. By definition, minor changes do not require preapproval by the FDA; instead, these can be handled by notification supplements.

Category 3 represents compounds with properties that generally preclude their development as drugs. Because they lack both solubility and permeability the formulator will be required to overcome one or the other of these problems before a drug with consistent absorption can be expected. This is probably the greatest formulation challenge of these drugs. Dissolution data for such compounds is not expected to be predictive of in-vivo performance. Subsequent changes in the manufacturing scale, method of manufacture or a formulation change outside of the "minor changes" defined by the Workshop Report will require a bioequivalence study.

Many of today's pharmaceutical products fall into category 2 and present unique challenges to the pharmaceutical scientist. Drugs with low solubility/high permeability may provide more frequent correlation of in-vitro and in-vivo dissolution than those with high sol- ubiliy/low permeability since the a priori asumption is that permeability is the more critical of these two measures.

In order to be assured that the drug product is performing reproducibly during scale-up, the dissolution profile of the drug product should be thoroughly investigated and contrasted to earlier (smaller) batches. It is suggested that the profile be determined (e.g.,15, $30,45,60,120$ and $180 \mathrm{~min}$.) until either $90 \%$ is dissolved or an asymptote is reached. Profiling should be done in multiple dissolution media in order to characterize the $\mathrm{pH}$ susceptibility of the drug product. Suggested media include water, $0.1 \mathrm{~N}$ $\mathrm{HCl}$, and USP buffers at $\mathrm{pH}$ $4.5,6.5$ and 7.5. In some cases, solubility aids may be required in order to provide dissolution that best correlates with in-vivo dissolution. Hopefully, one of these procedures will allow for correlation with a deconvoluted plasma concentration time curve. If so, subsequent changes to the formula or manufacturing process that result from scale-up can be justified by comparing the similarity of the dissolution profiles before and after scale-up.

\section{Concluding Remark}

It is important to note that the development of what has been called a 'biorelevant' dissolution method and specification(s) is product/formula specific and not an inherent property of a drug substance. 
QUALITY, continued from p. 3

Therefore, a major change in the formula or dosage form type will require the establishment of a new set of data to prove correlation of the dissolution method with the pharmacokinetic or pharmacodynamic properties of the dosage form.

\section{References:}

1. Immediate Release Scale-up and Post Approval Change (SUPAC)

Expert Working Group of the Chemistry Manufacturing Controls Coordinating Committee (CMC CC) of the Center for Drug Evaluation and Research of the Food and
Drug Administration . IN IGUIDE.IR9, November 29, 1994. Federal Register, 59 (237) 6409464096, (12/12/94).

2. J. P. Skelly, G. A. Van Buskirk, et al., Pharm. Res., 10 (2), 313-316, (1993).

3. J. P. Skelly, G. A. Van Buskirk, et al., Pharm. Res., 10 (12), 1800 1805, (1993).

4. J. P. Skelly, G. A. Van Buskirk, et al., J. Parenter. Sci. Technol., 75 (2), 52-56, (1993).

5. J. P. Skelly, G. A. Van Buskirk, et al, J. Parenter. Sci. Technol., 48 (2), 95-101, (1994).

6. J. P. Skelly, G. A. Van Buskirk, et al., Pharm. Technol., 19 (4), 6874, (1995).
7. J. P. Skelly, G. A. Van Buskirk, et al., Pharm. Technol., 19 (5), 4654, (1995).

8. J. P. Skelly, G. A. Van Buskirk, et al., Eur. J. Pharm. Biopharm., 39 (1), 40-43, (1993).

9. J. P. Skelly, G. A. Van Buskirk, et al., Eur. J. Pharm. Biopharm., 39 (4), 162-167, (1993).

10. G. L. Amidon, H. Lennernäs, et. al., Pharm. Res., 12 (3), 413 420, (1995). 